\title{
On the function "sandwiched" between $\alpha(G)$ and $\bar{\chi}(G)$
}

\author{
V. Y. Dobrynin ${ }^{1}$
}

Submitted: July 18, 1997; Accepted: September 2, 1997

\begin{abstract}
A new function of a graph $G$ is presented. Say that a matrix $B$ that is indexed by vertices of $G$ is feasible for $G$ if it is real, symmetric and $I \leq B \leq I+A(G)$, where $I$ is the identity matrix and $A(G)$ is the adjacency matrix of $G$. Let $\mathcal{B}(G)$ be the set of all feasible matrices for $G$, and let $\bar{\chi}(G)$ be the smallest number of cliques that cover the vertices of $G$. We show that

$$
\alpha(G) \leq \min \{\operatorname{rank}(B) \mid B \in \mathcal{B}(G)\} \leq \bar{\chi}(G)
$$

and that $\alpha(G)=\min \{\operatorname{rank}(B) \mid B \in \mathcal{B}(G)\}$ implies $\alpha(G)=\bar{\chi}(G)$.
\end{abstract}

The well known Lovász number $\vartheta(G)$ of a graph $G[1]$ is "sandwiched" between the size of the largest stable set in $G$ and the smallest number of cliques that cover the vertices of $G$

$$
\alpha(G) \leq \vartheta(G) \leq \bar{\chi}(G) .
$$

Some alternative definitions of $\vartheta(G)$ are introduced in [2][3] . For example,

$$
\begin{aligned}
\vartheta(G)=\max \{ & \Lambda(B) \mid B \text { is a real positive semidefinite matrix } \\
& \text { indexed by vertices of } G, \\
& B_{v v}=1 \text { for all } v \in V(G), \\
& \left.B_{u v}=0 \text { whenever } u-v \text { in } G\right\},
\end{aligned}
$$

where $\Lambda(B)$ is the maximum eigenvalue of $B, V(G)$ - the set of vertices of $G, u-v$ denotes the adjacency of vertices $u$ and $v$.

Call the matrix $B$ indexed by vertices of $G$ feasible for $G$ if

$$
\begin{aligned}
& B \text { is real and symmetric, } \\
& B_{v v}=1 \text { for all } v \in V(G), \\
& B_{u v}=0 \text { whenever } u+v \text { in } G, \\
& 0 \leq B_{u v} \leq 1 \text { whenever } u-v \text { in } G .
\end{aligned}
$$

Let $\mathcal{B}(G)$ be the set of all feasible matrices for $G$. Then [4]

$$
\bar{\chi}(G)=\min \left\{\operatorname{rank}(B) \mid B \in \mathcal{B}(G), B=C^{T} C, C \geq 0\right\},
$$

where the inequality denotes componentwise inequality.

The aim of this paper is to study a new function of graph $G$ 
Theorem. For all graphs $G$

$$
\alpha(G)=\min \{\operatorname{rank}(B) \mid B \in \mathcal{B}(G)\}
$$

implies $\alpha(G)=\bar{\chi}(G)$.

Proof. Let $S=\left\{v_{1}, v_{2}, \ldots, v_{\alpha(G)}\right\}$ be the stable set of $G, \bar{S}=V(G) \backslash S$ and $B \in \mathcal{B}(G)$ is a matrix such that $\operatorname{rank}(B)=\alpha(G)$. We can assume that

$$
B=\left(\begin{array}{cc}
I_{\alpha(G)} & X \\
X^{T} & Y
\end{array}\right)
$$

where $I_{\alpha(G)}$ is the identity $\alpha(G) \times \alpha(G)$-matrix.

Applying block Gauss elimination, $B$ reduces to the matrix

$$
B^{\prime}=\left(\begin{array}{cc}
I_{\alpha(G)} & X \\
0 & Y-X^{T} X
\end{array}\right)
$$

We have

$$
Y-X^{T} X=0
$$

or

$$
Y_{u v}-\sum_{w \in S} X_{w u} X_{w v}=0, \quad u, v \in \bar{S}
$$

since $\operatorname{rank}\left(B^{\prime}\right)=\operatorname{rank}(B)=\alpha(G)$.

Equation (1) gives us further information about the graph $G$.

(i) If $v \in \bar{S}$ then exists $u \in S$ such that $u-v$. Indeed, $Y_{v v}=1$ and $X_{w v} \geq 0$ for all $w \in S$. Hence, $\sum_{w \in S} X_{w v}^{2}=1$ and $X_{u v}>0$ for some $u \in S$.

(ii) If $v^{\prime}, v^{\prime \prime} \in \bar{S}$ and $X_{u v^{\prime}} X_{u v^{\prime \prime}}>0$ for some $u \in S$ then $v^{\prime}-v^{\prime \prime}$. Indeed, if $\sum_{w \in S} X_{w v^{\prime}} X_{w v^{\prime \prime}}>0$ then $Y_{v^{\prime} v^{\prime \prime}}>0$.

Let

$$
V_{u}=\{u\} \cup\left\{v \mid v \in \bar{S}, X_{u v}>0\right\}
$$

for all $u \in S$ and $G\left(V_{u}\right)$ be the subgraph induced from $G$ by leaving out all vertices except vertices from $V_{u}$. We know from (i) and (ii) that $G\left(V_{u}\right)$ is a clique and $V(G)=\cup_{u \in S} V_{u}$. Hence, $\bar{\chi}(G)=$ $\alpha(G)$.

Corollary. If $\bar{\chi}(G) \leq \alpha(G)+1$ then

$$
\bar{\chi}(G)=\min \{\operatorname{rank}(B) \mid B \in \mathcal{B}(G)\}
$$

For example, consider the Petersen graph $G$. We have $\alpha(G)=\vartheta(G)=4, \bar{\chi}(G)=5$. Hence, $\min \{\operatorname{rank}(B) \mid B \in \mathcal{B}(G)\}=5$.

There is a graph $G$ such that

$$
\alpha(G)<\min \{\operatorname{rank}(B) \mid B \in \mathcal{B}(G)\}<\bar{\chi}(G)
$$

Let $V(G)=2^{\{1,2,3,4,5,6\}}$ and $u-v$ iff $2 \leq|(u \backslash v) \cup(v \backslash u)| \leq 5$ for all $u, v \in V(G)$. Then [5] $\chi(G)=32$ and $\operatorname{rank}(A(G))=29$ where $A(G)$ is the adjacency matrix of $G$. Then $\bar{\chi}(\bar{G})=32$ and 
and

$$
\alpha(\bar{G})<\min \{\operatorname{rank}(B) \mid B \in \mathcal{B}(\bar{G})\}
$$

by theorem.

\section{Acknowledgement.}

The author is grateful to the anonymous referee for valuable remarks.

\section{References}

1. L. Lovász, "On the Shannon capacity of a graph", IEEE Transactions on Information Theory IT-25 (1979), 1-7.

2. Martin Grötschel, László Lovász, and Alexander Schrijver, Geometric Algorithms and Combinatorial Optimization (Springer-Verlag, 1988).

3. Donald E.Knuth, "The Sandwich Theorem", Electronic J. Combinatorics 1, A1 (1994), 48 pp.

4. V.Y.Dobrynin, "The chromatic number of a graph and rank of matrix associated with it", Vestnik Sankt-Peterburgskogo Universiteta, Ser.1, Issue 2, Vol.15 (1995), 120-122.

5. N.Alon, P.D.Seymour. "A counterexample to the rank-coloring conjecture", Journal of Graph Theory 13 (1989), 523-525. 